\title{
From Zero to Neuro-Reprogramming: Innovations in Translational Neuroregenerative Medicine
}

\author{
Ahmad Galuta ${ }^{1}$, Eve Tsai ${ }^{1,2,3}$ \\ ${ }^{1}$ Department of Neurosciences, Faculty of Medicine, University of Ottawa, Ontario, Canada \\ ${ }^{2}$ Neuroscience Program, Ottawa Hospital Research Institute, Ontario, Canada \\ ${ }^{3}$ Division of Neurosurgery, Department of Surgery, The Ottawa Hospital, Ontario, Canada
}

\section{ABSTRACT}

The acquisition of live human nervous tissue for research presents ethical and technical constraints. As a result, clinicians and scientists resort to using animal models to investigate human neuronal development and degeneration. However, innate differences in neurobiology between species impede the translation of disease pathologies and development of therapeutic strategies. The discovery and examination of endogenous neural stem cells (NSCs) have been critical for our understanding of neuronal development, degeneration, and regeneration. NSCs can exist in different developmental stages, embryonic through adult, and possess the capacity to generate the various cells that make up the nervous system. Human somatic cells can be obtained non-invasively and genetically reprogrammed into NSCs, serving as an alternative and ethical means to acquire stem cells for translational study and potential therapy. Novel methods to generate NSCs of various developmental origins and regional identities are evolving rapidly to provide safer, quicker, and more efficient genetic reprogramming strategies. Reprogrammed NSCs share many molecular and functional attributes with their endogenous counterparts and can be used for in vitro modeling at a large scale. The accessibility to study patient-specific NSCs allows for causal inferences of human disease mechanisms that may be unfeasible to model in animals. Given the novelty of this burgeoning field, the opportunity for translational discoveries in neuroregenerative medicine is unprecedented. This review will highlight the advances in manufacturing NSCs and their translational implications for disease modeling and potential treatment in the human nervous system.

$$
\text { RÉSUMÉ }
$$

Obtenir de tissu nerveux humain vivant pour la recherche présente des contraintes éthiques et techniques. Ainsi, les cliniciens et les scientifiques finissent par utiliser des modèles animaux pour étudier le développement et la dégénération neuronaux humains. Par contre, des différences innées entre les espèces en neurobiologie entravent la traduction de pathologies médicales et le développement de stratégies thérapeutiques. La découverte des cellules souches neurales (CSN) endogènes et leur étude ont été critique pour notre compréhension du développement, de la dégénération et de la régénération neuronaux. Les CSNs peuvent exister en différents stades de développement, embryonnaire à adulte, et possèdent la capacité de générer les différentes cellules qui froment le système nerveux. De plus important, les cellules somatiques humaines peuvent être obtenues de manière non invasive et reprogrammées génétiquement en CSNs, et ainsi peuvent se servir comme un moyen alternatif et éthique d'acquérir des cellules souches pour l'étude translationelle et des thérapies potentielles. De nouvelles méthodes pour générer des CSNs de plusieurs origines développementales et d'identités régionales évoluent rapidement pour fournir des stratégies génétiques de reprogrammation sécuritaire, rapides et efficaces. Les CSNs reprogrammées partagent plusieurs attributs moléculaires et fonctionnels avec leurs équivalents endogènes et peuvent être utilisées comme modélisation in vitro sur une grande échelle. L'accessibilité à étudier des CSNs propres aux patients permet l'ingérence causale de mécanismes de maladies humaines qui seront peut-être impossibles avec des modèles animaux. Malgré la nouveauté de ce champ naissant, i y a une opportunité sans précédent pour des découvertes translationelles en la médecine neuro-génératrice. Cette revue surlignera les avancements en la fabrication de CSNs et leur implication translationelles pour la modélisation de maladies et les traitements potentiels du système nerveux.

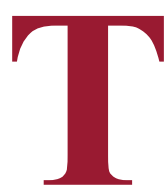

he acquisition of live human nervous tissue for research presents ethical and technical constraints. As a result, clinicians and scientists resort to using animal models to investigate human neuronal development and degeneration. However, innate differences in neurobiology between species impede the translation of disease pathologies and development of therapeutic strategies. The discovery and examination of

Keywords: Genetic Reprogramming; Neural Stem Cells; Translation; Neurodevelopment; Neurodegeneration 
endogenous neural stem cells (NSCs) have been critical for our understanding of neuronal development, degeneration, and regeneration. NSCs can exist in different developmental stages, embryonic through adult, and possess the capacity to generate the various cells that make up the nervous system. Human somatic cells can be obtained non-invasively and genetically reprogrammed into NSCs, serving as an alternative and ethical means to acquire stem cells for translational study and potential therapy. Novel methods to generate NSCs of various developmental origins and regional identities are evolving rapidly to provide safer, quicker, and more efficient genetic reprogramming strategies. Reprogrammed NSCs share many molecular and functional attributes with their endogenous counterparts and can be used for in vitro modeling at a large scale. The accessibility to study patient-specific NSCs allows for causal inferences of human disease mechanisms that may be unfeasible to model in animals. Given the novelty of this burgeoning field, the opportunity for translational discoveries in neuroregenerative medicine is unprecedented. This review will highlight the advances in manufacturing NSCs and their translational implications for disease modeling and potential treatment in the human nervous system.

\section{DISCOVERY OF ENDOGENOUS NSCS AND DEATH OF A DOGMA}

Throughout much of the 20th century, it was believed that the adult mammalian central nervous system had very little capacity to regenerate and fix itself. This dogma was set forth by the "father of neuroscience", Santiago Ramón y Cajal, famous for his intricate drawings of neuronal networks that established the neuron doctrine (1). Through his observations of severed neuronal axons, he stated, "In adult centres the nerve paths are something fixed, ended, immutable. Everything may die, nothing may be regenerated. It is for the science of the future to change, if possible, this harsh decree" (2). However, nearing the end of the 20th century, the discovery of endogenous neural stem cells (NSCs) in the brain and spinal cord shattered this long-held belief (2-4). Today, human NSCs are being manufactured "from scratch" and used for modeling human neuronal developmental and degeneration as well as personalized drug screening. They are also being tested as therapeutic agents for treatment (5-8).

Both the brain and spinal cord host NSCs within specialized compartments in the ventricular regions as well as the dentate gyrus of the hippocampus. NSCs are defined by their ability to generate specialized cell types of the nervous system including neurons, oligodendrocytes, and astrocytes and thus pose as prime candidates for cellular replacement of damaged, diseased, or dead cells in a variety of neurodegenerative contexts (10). As such, endogenous NSCs have been subject to targeted manipulation in animal models to promote regeneration (8,11-13). However, endogenous NSCs do not play a significant role in physiological central nervous system repair which partly contributed to the scientific community's dismissal of them throughout the 20th century. Moreover, their numbers in vivo are limited and spatially restricted, which may be a concern when the generation of enough cells in the right location is a requirement for effective repair (15-17).

\section{A NEW ERA: GENETICALLY REPROGRAMMED NSCS}

To circumvent issues with targeting endogenous NSCS, methods have been developed to manufacture NSCs that resemble endogenous NSCs at various developmental stages and from different regions in the nervous system (1-4). These revolutionary advancements have provided researchers with unprecedented access to study human neural cells with few ethical and technical constraints. Notably, reprogrammed NSCs have been used to treat neurological disorders in preclinical animal models and have entered clinical testing in the treatment of Parkinson's disease, spinal cord injuries, and macular degeneration of the eye (5-7). Since its inception in 2006, the field of NSC reprogramming has rapidly expanded without any signs of slowing down (1,9-11). The remainder of this review will highlight the technological advancements in NSC reprogramming methods as well as their value in translational research and clinical use.

\section{IN VITRO REPROGRAMMING: GENOMIC, PROTEOMIC OR CHEMICAL INTERVENTIONS}

The most common means for genetically reprogramming NSCs involves an in vitro intervention. First, a small patient sample of skin, urine, hair, or blood is obtained and cultured to generate enough cells (e.g. fibroblasts, keratinocytes, mesenchymal cells) as starting material. Subsequently, the global gene expression profile of the starting population must be altered to match that of NSCs which can be achieved via the induction of gene regulatory networks (GRNs) characteristic of NSCs (12-15). Put simply, expression of certain transcription factors dubbed "pioneering factors" are sufficient to orchestrate a change in the gene expression landscape of the whole cell by activating such GRNs. For example, induced overexpression of Sox 2 alone is capable of converting somatic cells into NSCs both in vitro and in vivo (next section) $(16,17)$. In this case, Sox 2 binds to DNA in regions of heterochromatin that are normally 
inaccessible, resulting in the upregulation of NSC-specific genes and downregulation of the original cell identity. The induced NSCs (iNSCs) produced are capable of extensive selfrenewal and multi-potential differentiation; they also exhibit gene expression profiles characteristic to NSCs (18). Therefore, at the fundamental level, iNSCs possess similar molecular and functional properties as endogenous NSCs.

To induce expression of pioneering factors, several strategies have been used including genomic, proteomic, and chemical interventions $(1,12)$. The most effective and commonly used strategy is the integration and forced overexpression of pioneering factor genes using viruses (19). This strategy permits the stable expression of transduced genes in dividing daughter cells but comes with the risk of DNA instability due to random gene insertion. As such, iNSCs generated using this method are not clinically translatable. However, nontransducing strategies have been developed to minimize the risks associated with permanent genomic modifications. These strategies include non-transducing viruses (e.g. adenovirus, Sendai virus), transient overexpression of pioneering factor genes using non-viral methods (e.g. electroporation and transfection of plasmids or RNA), transfection with transcription factor proteins, and/or using small chemical molecules to modulate endogenous transcriptional machinery (e.g. histone modifying proteins) $(1,12,13)$. The latter strategy, when using only small chemical molecules, is dubbed the safest method for clinical translation because it avoids the introduction of foreign genetic material. Therefore, more protocols utilizing this strategy are being developed $(15,20)$.

Despite low reprogramming efficiencies of most methods, once iNSCs are formed, they can be expanded long-term in culture to scale production for downstream applications $(12,19,21)$. iNSCs can also be cryo-preserved to be shipped and used for later applications with no decline in regenerative potential. This allows enough iNSCs to be generated for autologous transplant or creation of a cell bank for allogenic transplantation (8). However, given the various methods of generating iNSCs, it is unclear if iNSCs can reliably give rise to all major cell types of the nervous system as similarly as their endogenous counterparts. Hence, there is continued interest in the field for generating iNSCs that bear the utmost homology to endogenous NSCs. Furthermore, it has been demonstrated that endogenous NSCs and iNSCs of spinal cord identity are superior for the repair of spinal cord injury compared to their brain counterparts $(22,23)$. Therefore, it is expected that iNSCs will be most therapeutically effective when placed in their "natural" environment which they can recognize and support.

\section{IN VIVO REPROGRAMMING OF ASTROCYTES}

In 2013, in vivo reprogramming of rodent spinal cord astrocytes into iNSCs was first reported which was replicated in the rodent brain $(17,24,25)$. Astrocytes are abundant in the nervous system making them an attractive target for cellular reprogramming (26). It must be noted that other cell types can be reprogrammed into NSCs in vivo, however, given their relative abundance, astrocytes may be the best candidate. Furthermore, relative to the cells commonly used in in vitro reprogramming, the gene expression profile of astrocytes more closely resembles NSCs which facilitates genetic reprogramming. In vivo reprogrammed iNSCs display characteristic self-renewal, multi-potential differentiation, and can differentiate into neurons which integrate and form synaptic connections with neighbouring neurons. Importantly, iNSCs can be reprogrammed in neurodegenerative disease and injury models including Alzheimer's, brain stab injury, and spinal cord injury and can survive long-term (up to 8 months) indicating the therapeutic potential of this strategy $(17,24,27)$. However, an impediment to effective regeneration lies in the ability to faithfully direct iNSC fate in vivo and generate enough cells with a desired specialized phenotype.

To selectively reprogram astrocytes in vivo, a viral-based intervention is commonly used to induce overexpression of pioneering factor Sox2 (Figure 1) $(17,27,28)$. Some main advantages of directly converting cells in vivo include that it avoids the time constraints imposed by in vitro reprogramming and is potentially less invasive. However, a safe and efficient means for the viral delivery of genetic material will be important for realization of this strategy in humans. Such a strategy must also meet the need for targeting regional populations of astrocytes, thereby allowing for a mechanistic approach for directing regeneration in a spatial manner while minimizing off-target effects (Figure 1) (27).

\section{MODELING NEURONAL DEVELOPMENT AND DEGENERATIVE DISEASES}

Reprogrammed NSCs have provided unprecedented access to study live human brain and spinal cord neuronal cells, which were previously only attainable through biopsies, postmortem tissue, or embryonic derived tissue. Since iNSCs can be directed to a specific developmental lineage from embryonic to adult, appropriate models for neurodevelopment and neurogenesis can be generated (Figure 2) $(2,29,30)$. Furthermore, patient-derived iNSCs can be generated which 
recapitulate characteristic hallmarks of the associated disease including amyotrophic lateral sclerosis, multiple sclerosis, Alzheimer's, Parkinson's, and Huntington's disease (30-36). For example, iNSC-derived neurons from Alzheimer's patients display the typical pathological features in vitro including elevated amyloid beta plaques and phosphorylated tau proteins $(2,32)$. Similarly, neurons derived from Parkinson's patients posses higher than normal levels of both oxidative stress and alpha-synuclein impairing neuronal survival and function $(37,38)$. Subsequent interventions using molecular or genetic techniques can then be applied to rescue pathological phenotypes in patient cells or induced in control cells which do not bear the original disease phenotype $(2,30,31,39,40)$. Therefore, iNSCs represent a new platform for which studies can be designed to better understand human specific disease mechanisms and facilitate the development of therapeutic targets.

In addition to drug development, iNSCs can be used for drug screening to predict whether a patient will be drug-resistant as is the case with many neuropsychiatric conditions $(4,33,39,41)$. An interesting study involving iNSC-derived hippocampal granule neurons from patients with bipolar disorder demonstrated hyperexcitability, irregular mitochondrial function and altered gene expression compared to healthy controls. The impaired phenotypes in patient derived neurons were rescued with lithium treatment but only in the subset of patients who responded to treatment. Gene expression analysis revealed that lithium significantly altered gene expression in neurons derived from lithium-responsive patients (560 genes) compared to lithium-nonresponsive patients (40 genes) (42). This presents an opportunity for personalized drug screening, as patient specific responses to drugs can be observed in vitro while also determining the genetic predispositions of drug resistance. As such, efforts to model cellular mechanisms of disease have increased and represent important tools for personalized medicine and potential development of therapeutic treatments in non-responsive patients.

An emerging strategy to model human brain development and dysfunction involves the formation of "brain organoids" from iNSCs $(4,30,39-41,43-45)$. These in vitro miniature brains are developed in 3D and can consist of distinct human brain regions including ventricles, cerebral cortex, hippocampus, hypothalamus, forebrain, midbrain, choroid plexus, and more. Brain organoids can be generated either by the autonomous self-regulating activity of iNSCs to form random brain structures or by directing the development of iNSCs with signaling cues to form distinct structures. Furthermore, different regionalized brain organoids can be fused ("assembloids") to model brain regional connectivity and interactions. For example, inhibitory GABAergic interneurons migrate from the ventral forebrain into the dorsal cortex during normal brain development which can be recapitulated in assembloids consisting of ventralized and dorsalized regions (46).

The main advantage of $3 \mathrm{D}$ organoid modeling is that it permits cell-to-cell communication among the different specialized cells in the organoid, thereby mimicking in vivo brain interactions more accurately than $2 \mathrm{D}$ in vitro systems. The intrinsic complexity of this model is useful to study the interactions amongst different human brain cells, immune cells, and pathogens, which is necessary for a wholesome understanding of pathophysiology. Moreover, these brain structures are reminiscent of human-specific neurodevelopment (e.g. outer radial glial cells in cerebral
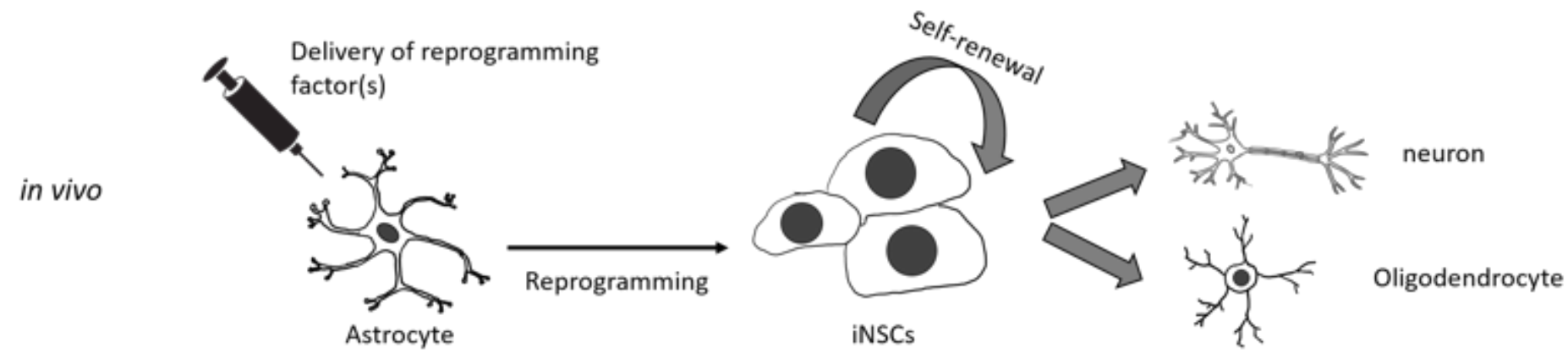

Figure 1. Method and rationale for astrocyte reprogramming into iNSCs in vivo. Astrocytes are selectively targeted using viral vectors and induced to overexpress reprogramming factors. Resulting iNSCs can self-renew and differentiate into neurons and oligodendrocytes which functionally integrate with the existing neuroanatomy. Challenges remains to safely and selectively target regional astrocyte populations as well as to direct fate of iNSCs towards specific neuronal and oligodendrocyte lineages. 
cortex development) which is not possible to study in animal models (47). Therefore, human brain organoids are better suited than animal models for the study of human-specific neurodevelopmental disorders such as micro/macrocephaly $(48,49)$.

Neuropsychiatric disorders, such as schizophrenia and autism spectrum disorder, have also been difficult to model in animals given their polygenic basis and complex pathophysiology involving dysregulation in cell-to-cell communication and brain circuitry $(33,39,41,50,51)$. The nature of brain organoids is such that neural circuitry is integrated within the system. Therefore, neural transmission among regionalized brain organoids can be causally dissected using molecular and genetic manipulations in conjunction with electrophysiological analysis. Another major advantage is that organoids from patients and family-matched controls can be studied in comparison to one another to reveal significant genetic contributors to disease pathology (Figure 2) $(52,53)$. (Poly)genetic mutations present as defects in organoid development, cell differentiation and maturation, neuronal synapse function, and circuit transmission. A major limitation of this model, however, is the furthest size and developmental stage that organoids can attain before becoming necrotic. Engineering organoids to contain vasculature will permit further development and maturation in vitro for modeling post-natal brain development and degeneration $(45,54)$.
Finally, it is crucial to integrate the information derived from organoid research with in vivo neurophysiology for reliable and accurate inference. This should be addressed by complementing in vitro modeling with live human and animal studies.

\section{CLINICAL APPLICATION}

The human central nervous system is ineffective at functional recovery over the course of degeneration or injury and thus may benefit from an exogenous source of regeneration (55-57). iNSCs are a promising tool for the treatment of neurodegenerative disorders, given they are inherently programmed to regenerate the different specialized cells of the nervous system (18). As such, iNSCs might be able to replace degenerating and/or dead cells to combat the progression of neurodegeneration and restore homeostatic neuronal functioning $(18,23,58)$. Pre-clinical testing involving implantation of NSC grafts in animals has proven to be beneficial for multiple conditions $(59,60)$. Some of the successful animal trials progressed towards clinical trials for Parkinson's disease, spinal cord injury, and macular degeneration of the eye (5-7). However, these trials are still in their infancy and their effectiveness has yet to be determined. Major challenges for these strategies include promoting cell graft survival, controlling iNSC fate in vivo, and promoting structural and functional integration of iNSCs with host cells.

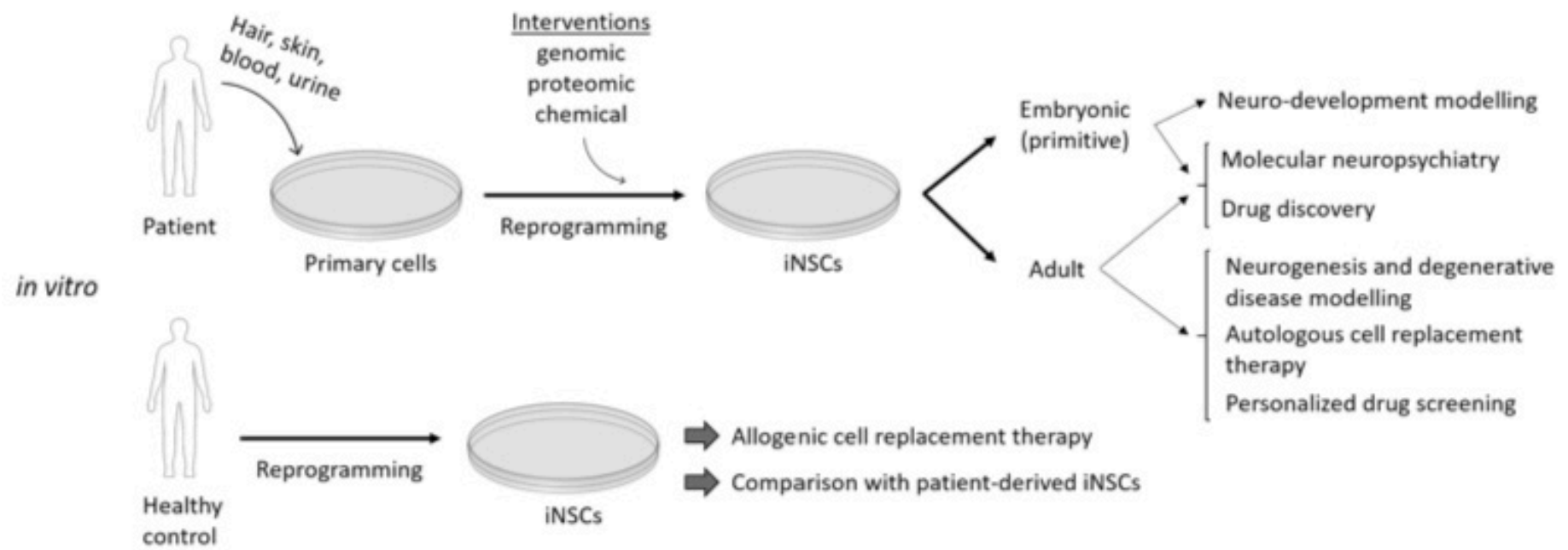

Figure 2. Overview of genetic reprogramming procedure in vitro. and translational implications of iNSCs.

(A) First, patient somatic cells are obtained and (B) cultured to yield enough primary cells for reprogramming. (C) Many methods including genomic, proteomic or chemical interventions are available for reprogramming iNSCs with specified developmental and regional identities. iNSCs have multiple translational purposes including cell replacement therapy and discovery of human specific neurodevelopment and disease mechanisms. (D) iNSCs can be generated from control patients and used for allogenic transplants or comparative analysis with patient-derived iNSCs. and oligodendrocyte lineages. 
Nonetheless, the development of iNSCs from adult human tissue has mitigated the ethical constraints that accompany embryonic and fetal-derived tissue use while also providing an autologous source of cells for transplant which minimizes immunogenicity (61). Therefore, iNSCs can be patient-specific and scaled up in production for later transplant. The obvious drawback is the time frame during which iNSCs need to be cultured in vitro, thereby representing a possible limitation for patients that require an urgent intervention. A solution could be to use an isogenic cell bank for all patients, which would permit immediate access to cells and minimize the variability between different iNSC lines. This, however, would replace the benefits of autologous treatment with the risks of allogenic transplantation $(8,61)$.

\section{CONCLUSION}

Insult to the human central nervous system was presumed to be permanent, but today's scientific advances in genetic reprogramming have revolutionized the way we study and treat neurological disorders. The advancements in genetic reprogramming strategies to create iNSCs has provided scientists with human neuronal cells and organoids that mimic patient-specific conditions and can be used to identify the molecular underpinnings of human disease. This strategy can be used to develop therapeutic targets and test drug efficacy in a patient-specific manner. Given their endogenous regenerative capacity and demonstrated effectiveness in pre-clinical models, iNSCs hold the potential for treating a variety of neurodegenerative disorders. The major challenges moving forward include creating more developed in vitro models of human neurobiology, integrating the information from in vitro models with our understanding of in vivo neurophysiology, and translating the pre-clinical effectiveness of iNSC transplantation into successful human trials.

\section{ACKNOWLEDGEMENTS}

We would like to thank and acknowledge Ryan Sandarage, Jason C.S. Kwan, and Alexander Sanghwan for their contribution to and revision of this manuscript.

\section{REFERENCES}

1. Shahbazi E, Mirakhori F, Ezzatizadeh V, Baharvand H. Reprogramming of somatic cells to induced neural stem cells. Vol. 133, Methods. Elsevier Inc.; 2018. p. 21-8.

2. Hou PS, Chuang CY, Yeh CH, Chiang W, Liu HJ, Lin TN, et al. Direct Conversion of Human Fibroblasts into Neural Progenitors Using Transcription Factors Enriched in Human ESC-Derived Neural Progenitors. Stem Cell Reports. 2017;8(1):54-68.

3. Ogura T, Sakaguchi H, Miyamoto S, Takahashi J. Three-dimensional induction of dorsal, intermediate and ventral spinal cord tissues from human pluripotent stem cells. Development. 2018;145(16):dev162214.

4. Wang H. Modeling Neurological Diseases With Human Brain Organoids. Front Synaptic Neurosci. 2018;10(15):1-14.

5. Barker RA, Parmar M, Studer L, Takahashi J. Human Trials of Stem CellDerived Dopamine Neurons for Parkinson's Disease: Dawn of a New Era. Cell Stem Cell. 2017 Nov;21(5):569-73.

6. Li F, Hu J, He TC. iPSC-based treatment of age-related macular degeneration (AMD): The path to success requires more than blind faith. Genes Dis. 2017;4(2):41-2.

7. lida T, Okano H, Sugai K, Yamaguchi R, Isoda M, Ohkubo T, et al. Concise Review: Laying the Groundwork for a First-In-Human Study of an Induced Pluripotent Stem Cell-Based Intervention for Spinal Cord Injury. Stem Cells. 2018;37(1):6-13.

8. Morizane A, Doi D, Kikuchi T, Okita K, Hotta A, Kawasaki T, et al. Direct comparison of autologous and allogeneic transplantation of IPSC-derived neural cells in the brain of a nonhuman primate. Stem Cell Reports. 2013;1(4):283-92.

9. Li G, Che MT, Zhang K, Qin LN, Zhang YT, Chen RQ, et al. Graft of the NT-3 persistent delivery gelatin sponge scaffold promotes axon regeneration, attenuates inflammation, and induces cell migration in rat and canine with spinal cord injury. Biomaterials. 2016;83:233-48.

10. Yamashita T, Abe K. Recent Progress in Cell Reprogramming Technology for Cell Transplantation Therapy. Neurol Med Chir (Tokyo). 2016;56(3):97101.

11. Takahashi K, Yamanaka S. Induction of Pluripotent Stem Cells from Mouse Embryonic and Adult Fibroblast Cultures by Defined Factors. Cell. 2006;126(4):663-76.

12. Xu J, DuY, Deng H. Direct lineage reprogramming: Strategies, mechanisms, and applications. Cell Stem Cell. 2015;16(2):119-34.

13. Gopalakrishnan S, Hor P, Ichida JK. New approaches for direct conversion of patient fibroblasts into neural cells. Brain Res. 2017;1656:2-13.

14. Jopling C, Boue S, Belmonte JCl. Dedifferentiation, transdifferentiation and reprogramming: Three routes to regeneration. Nat Rev Mol Cell Biol. 2011;12(2):79-89.

15. Xie M, Tang S, Li K, Ding S. Pharmacological Reprogramming of Somatic Cells for Regenerative Medicine. Acc Chem Res. 2017;50(5):1202-11.

16. Julian LM, McDonald AC, Stanford WL. Direct reprogramming with SOX factors: masters of cell fate. Curr Opin Genet Dev. 2017;46:24-36.

17. Su Z, Niu W, Liu ML, Zou Y, Zhang CL. In vivo conversion of astrocytes to neurons in the injured adult spinal cord. Nat Commun. 2014;5:1-15.

18. Gage FH, Temple S. Neural stem cells: Generating and regenerating the brain. Neuron. 2013;80(3):588-601.

19. Malik N, Rao MS. A review of the methods for human iPSC derivation. In Methods in Molecular Biology. 2013. p. 23-33.

20. Zhang M, Lin YH, Sun YJ, Zhu S, Zheng J, Liu K, et al. Pharmacological reprogramming of fibroblasts into neural stem cells by signaling-directed transcriptional activation. Cell Stem Cell. 2016;18(5):653-67.

21. Omole $A E$, Fakoya $A O J$. Ten years of progress and promise of induced pluripotent stem cells: historical origins, characteristics, mechanisms, limitations, and potential applications. PeerJ. 2018;6:e4370.

22. Kadoya K, Lu P, Nguyen K, Lee-Kubli C, Kumamaru H, Yao L, et al. Spinal cord reconstitution with homologous neural grafts enables robust corticospinal regeneration. Nat Med. 2016;22(5):479-87.

23. Kumamaru H, Kadoya K, Adler AF, Takashima Y, Graham L, Coppola G, et al Generation and post-injury integration of human spinal cord neural stem cells. Nat Methods. 2018;15(9):723-31.

24. Guo Z, Zhang L, Wu Z, Chen Y, Wang F, Chen G. In vivo direct reprogramming of reactive glial cells into functional neurons after brain injury and in an Alzheimer's disease model. Cell Stem Cell. 2014;14(2):188-202.

25. Wolf DA, Bjorklund A, Lau S, Pereira M, Parmar M, Pfisterer U, et al. Generation of induced neurons via direct conversion in vivo. Proc Natl Acad Sci. 2013;110(17):7038-43.

26. Torper O, Götz M. Brain repair from intrinsic cell sources. Prog Brain Res. 2017;230:69-97.

27. Chen G, Wernig M, Berninger B, Nakafuku M, Parmar M, Zhang C-L. In Vivo 
Reprogramming for Brain and Spinal Cord Repair. eNeuro. 2015;2(5):1-6.

28. Zou Y, Vue TY, Bachoo R, Zhang C-L, Niu W, Zang T, et al. SOX2 Reprograms Resident Astrocytes into Neural Progenitors in the Adult Brain. Stem Cell Reports. 2015;4(5):780-94.

29. Ring KL, Tong LM, Balestra ME, Javier R, Andrews-Zwilling $Y$, Li G, et al. Direct reprogramming of mouse and human fibroblasts into multipotent neural stem cells with a single factor. Cell Stem Cell. 2012;11(1):100-9.

30. Di Lullo E, Kriegstein AR. The use of brain organoids to investigate neural development and disease. Nature Reviews Neuroscience. 2017;18:573-84.

31. Bordoni M, Fantini V, Pansarasa O, Cereda C. From Neuronal Differentiation of iPSCs to 3D Neural Organoids: Modeling of Neurodegenerative Diseases. Int J Mol Sci. 2018 Nov 5;19(12).

32. Fang $Y$, Gao T, Zhang B, Pu J. Recent advances: Decoding Alzheimer's disease with stem cells. Frontiers in Aging Neuroscience. 2018;10:77

33. Quadrato G, Brown J, Arlotta P. The promises and challenges of human brain organoids as models of neuropsychiatric disease. Nature Medicine. 2016;22:1220-8.

34. Devlin AC, Burr K, Borooah S, Foster JD, Cleary EM, Geti I, et al. Human iPSC derived motoneurons harbouring TARDBP or C9ORF72 ALS mutations are dysfunctional despite maintaining viability. Nat Commun. 2015;6:1-12.

35. Di Ruscio A, Patti F, Welner RS, Tenen DG, Amabile G. Multiple sclerosis: Getting personal with induced pluripotent stem cells. Cell Death and Disease. 2015;6:e1806.

36. Hou P-S, Chuang C-Y, Yeh C-H, Chiang W, Liu H-J, Lin T-N, et al. Stem Cell Reports Ar ticle Direct Conversion of Human Fibroblasts into Neural Progenitors Using Transcription Factors Enriched in Human ESC-Derived Neural Progenitors. Stem Cell Reports. 2017;8:54-68.

37. Funayama $M$, Kobayashi $T$, Okano $H$, Mochizuki H, Kosakai A, Amagai $M$, et al. Mitochondrial dysfunction associated with increased oxidative stress and a-synuclein accumulation in PARK2 PSSC-derived neurons and postmortem brain tissue. Mol Brain. 2012;5(1):35.

38. Azabdaftari G, Hu Z, Ren Y, Yuen EY, Nakaso K, Yan Z, et al. Parkin controls dopamine utilization in human midbrain dopaminergic neurons derived from induced pluripotent stem cells. Nat Commun. 2012;3:668.

39. LaMarca EA, Powell SK, Akbarian S, Brennand KJ.Modeling Neuropsychiatric and Neurodegenerative Diseases With Induced Pluripotent Stem Cells. Front Pediatr. 2018;6:82.

40. Brown J, Quadrato G, Arlotta P. Studying the Brain in a Dish: 3D Cell Culture Models of Human Brain Development and Disease. In: Current Topics in Developmental Biology. 2018. p. 99-122.

41. Amin ND, Paşca SP. Building Models of Brain Disorders with ThreeDimensional Organoids. Neuron. 2018;100:389-405.

42. Mertens J, Wang QW, Kim Y, Yu DX, Pham S, Yang B, et al. Differential responses to lithium in hyperexcitable neurons from patients with bipolar disorder. Nature. 2015;527(7576):95-9.

43. Lee CT, Bendriem RM, Wu WW, Shen RF. 3D brain Organoids derived from pluripotent stem cells: Promising experimental models for brain development and neurodegenerative disorders Julie Y.H. Chan. Journal of Biomedical Science. 2017;24:59.

44. Dutta D, Heo I, Clevers H. Disease Modeling in Stem Cell-Derived 3D Organoid Systems. Trends in Molecular Medicine. 2017;23:393-410.

45. Liu F, Huang J, Zhang L, Chen J, Zeng Y, Tang Y, et al. Advances in Cerebral Organoid Systems and their Application in Disease Modeling. Neuroscience. 2019;399:28-38.

46. Bagley JA, Reumann D, Bian S, Lévi-Strauss J, Knoblich JA. Fused cerebral organoids model interactions between brain regions. Nat Methods. 2017;14(7):743-51.

47. Lui JH, Kriegstein AR, Hansen D V, Parker PRL. Neurogenic radial glia in the outer subventricular zone of human neocortex. Nature. 2010;464(7288):554-61.

48. Renner M, Knoblich JA, Wenzel D, Lancaster MA, Hurles ME, Homfray T, et al. Cerebral organoids model human brain development and microcephaly. Nature. 2013;501(7467):373-9.

49. Muffat J, Omer A, Bosch I, Knoblich JA, Lancaster MA, Li Y, et al. Induction of Expansion and Folding in Human Cerebral Organoids. Cell Stem Cell. 2016;20(3):385-96.
50. Bramon E, Domenici E, Knight J, Lencz T, Liu J, McQuillin A, et al. Contribution of copy number variants to schizophrenia from a genomewide study of 41,321 subjects HHS Public Access Author manuscript. Nat Genet. 2017;49(1):27-35.

51. Ripke S, Neale BM, Corvin A, Walters JTR, Farh KH, Holmans PA, et al. Biological insights from 108 schizophrenia-associated genetic loci. Nature. 2014;511(7510):421-7.

52. Mariani J, Coppola G, Zhang P, Abyzov A, Provini L, Tomasini L, et al. FOXG1Dependent Dysregulation of GABA/Glutamate Neuron Differentiation in Autism Spectrum Disorders. Cell. 2015;162(2):375-90.

53. Ripke S, Neale BM, Corvin A, Walters JTR, Farh KH, Holmans PA, et al. Biological insights from 108 schizophrenia-associated genetic loci. Nature. 2014;511(7510):421-7.

54. Kelava I, Lancaster MA. Dishing out mini-brains: Current progress and future prospects in brain organoid research. Developmental Biology. 2016;420:199-209.

55. Grégoire C-A, Goldenstein BL, Floriddia EM, Barnabé-Heider F, Fernandes KJL. Endogenous neural stem cell responses to stroke and spinal cord injury. Glia. 2015;63(8):1469-82.

56. Becker CG, Becker T, Hugnot JP. The spinal ependymal zone as a source of endogenous repair cells across vertebrates. Prog Neurobiol. 2018;170(April):67-80.

57. Bergmann O, Spalding KL, Frisén J. Adult neurogenesis in humans. Cold Spring Harb Perspect Med. 2015:5(8):1-13.

58. Kikuchi T, Morizane A, Doi D, Magotani H, Onoe H, Hayashi T, et al. Human iPS cell-derived dopaminergic neurons function in a primate Parkinson's disease model. Nature. 2017;548(7669):592-6.

59. Koliatsos VE, Doughty ML, Azari H, Giordano James J, Wu S, FitzGerald KT, et al. On the Viability and Potential Value of Stem Cells for Repair and Treatment of Central Neurotrauma: Overview and Speculations. Front Neurol. 2018;1:602.

60. Mclauchlan D, Robertson NP. Stem cells in the treatment of central nervous system disease. J Neurol. 1234;265:984-6.

61. Angelos MG, Kaufman DS. Pluripotent stem cell applications for regenerative medicine. Curr Opin Organ Transplant. 2015;20(6):663-70. 